\title{
The sigma-meson exchange contribution to the muonic hy- drogen Lamb shift
}

\author{
A.E. Dorokhov ${ }^{1,2, *}$, A.P. Martynenko ${ }^{2}$, F.A. Martynenko ${ }^{2}$, and A.E. Radzhabov ${ }^{2,3}$ \\ ${ }^{1}$ Joint Institute of Nuclear Research, BLTP,141980, Moscow region, Dubna, Russia \\ ${ }^{2}$ Samara University, 443086, Samara, Russia \\ ${ }^{3}$ Matrosov Institute for System Dynamics and Control Theory SB RAS, 664033, Irkutsk, Russia
}

\begin{abstract}
The sigma $(\sigma)$ meson exchange contribution to the potential of the muon-proton interaction in muonic hydrogen induced by the $\sigma$-meson coupling to two photons is estimated. The transition form factor $\sigma \rightarrow \gamma \gamma$ is deduced from the quark model and experimental data on the decay widths $\Gamma_{\sigma \gamma \gamma}$. It is shown that scalar meson exchange contribution to the Lamb shift in muonic hydrogen, $\Delta E^{L s}(2 P-2 S)$, is rather large and relevant for a comparison with coming precise experimental data.
\end{abstract}

\section{Introduction}

Precise investigation of the Lamb shift and hyperfine structure of light muonic atoms is a fundamental problem for testing the Standard model and establishing the exact values of its parameters, test of bound state theory in QED, as well as searching for effects of new physics. At present, the relevance of these studies is related to recent experiments conducted by the collaboration CREMA (Charge Radius Experiments with Muonic Atoms) [1,2] with muonic hydrogen and ions of muonic helium by methods of laser spectroscopy. As a result of measuring the transition frequency $\left(2 P_{3 / 2}^{F=2}-2 S_{1 / 2}^{F=1}\right)$ a more accurate value of the proton charge radius was found to be $r_{p}=0.84184(67) \mathrm{fm}$, which is different from the value recommended by CODATA for $5 \div 7 \sigma$ [3]. The CODATA value is based on the spectroscopy of the electronic hydrogen atom and on electron-nucleon scattering data. The measurement of the transition frequency $\left(2 P_{3 / 2}^{F=1}-2 S_{1 / 2}^{F=0}\right)$ for the singlet $2 S$ of the state $(\mu p)$ provides the hyperfine splitting of the $2 S$ energy level in muonic hydrogen, and thus the values of the Zemach's radius $r_{Z}=1.082(37) \mathrm{fm}$ and magnetic radius $r_{M}=0.87(6) \mathrm{fm}$. The first measurement of three transition frequencies between energy levels $2 P$ and $2 S$ for muonic deuterium $\left(2 S_{1 / 2}^{F=3 / 2}-2 P_{3 / 2}^{F=5 / 2}\right),\left(2 S_{1 / 2}^{F=1 / 2}-2 P_{3 / 2}^{F=3 / 2}\right),\left(2 S_{1 / 2}^{F=1 / 2}-2 P_{3 / 2}^{F=1 / 2}\right)$ allowed to obtain in 2.7 times more accurate value of the charge radius of the deuteron, which is also less than the value recommended by CODATA [3], by $7.5 \sigma$. As a result, there is an inexplicable discrepancy between the values of such fundamental parameters, like the charge radius of a proton and deuteron, obtained from electronic and muonic atoms. In the process of searching for possible solutions of the proton charge radius "puzzle" the task of a more accurate theoretical construction of the particle interaction operator in quantum electrodynamics is discussed. The calculation of new corrections in the energy spectrum of muonic atoms acquires a special urgency.

\footnotetext{
*e-mail: dorokhov@theor.jinr.ru
} 
Among the various electromagnetic interactions, the processes of two-photon meson production take a special place. They have been studied experimentally for quite a long time (see for a review [4]). With the development of the quark model and nonperturbative methods of quantum chromodynamics, such reactions, as well as the reverse decay processes of mesons into two photons, were constantly in the field of intensive theoretical studies. New interest in $\gamma+\gamma \rightarrow$ meson processes is connected with their possible role as a new source of interactions between leptons and nucleons (see Fig. 1). The first estimates of the contribution of effective meson exchanges in muonic hydrogen, which have already appeared, show that this contribution can be significant [5-10].

In our previous papers $[9,10]$ we have investigated the role of effective exchanges of axial vector and pseudoscalar mesons for the position of the energy levels in muonic hydrogen. In this study we extend our analysis to the case of the lightest scalar $\sigma(550)$ meson. So, the numerical calculation of corresponding shifts in fine and hyperfine structure of muonic atoms looks as an important task which can shed light on the proton charge radius puzzle.

\section{Muon hydrogen potential via the $\sigma$-meson exchange}

The amplitude of the one-meson exchange between the muon and the proton arises as a result of the transition of two virtual photons into a scalar meson. The vertex function describing this process plays a central role in the study of meson exchange, since a good prediction of the magnitude of the shift in energy levels depends primarily on it. For the muon hydrogen the $\sigma$-meson exchange potential (Fig. 1) is given in momentum space as [11]

$$
V_{\sigma}(q)=\left(2 E_{k} 2 E_{k^{\prime}} 2 E_{p} 2 E_{p^{\prime}}\right)^{-1 / 2}\left[\bar{u}\left(k^{\prime}\right) \Gamma_{\sigma \mu \mu}\left(q^{2}\right) u(k)\right] \Delta_{\sigma}(q)\left[\bar{N}\left(p^{\prime}\right) \Gamma_{\sigma N N}\left(q^{2}\right) N(p)\right],
$$

where $k, k^{\prime}\left(p, p^{\prime}\right)$ are incoming and outgoing muon (proton) momenta, $q=k^{\prime}-k=p-p^{\prime}$ is the momentum transfer, $\Delta_{\sigma}(q)$ is the $\sigma$ meson propagator, and $\Gamma_{\sigma \mu \mu}, \Gamma_{\sigma N N}$ is the $\sigma$ meson vertex functions with muon and proton, respectively.

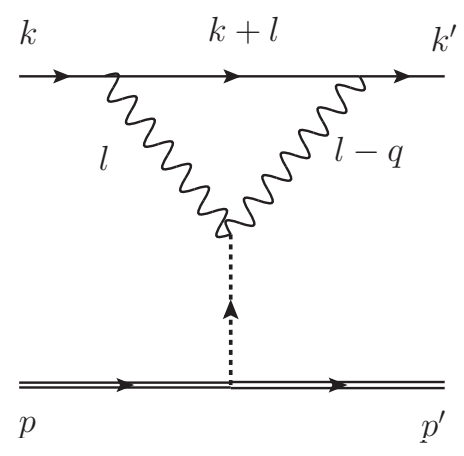

Figure 1. Muon-proton interaction induced by scalar meson exchange.

For the mass and width of the $\sigma$-meson we take

$$
m_{\sigma}=0.5 \mathrm{GeV}, \quad \Gamma_{\sigma}=0.33 \mathrm{GeV},
$$

which is approximately an average of the experimental values obtained at Fermilab E791 [12] and CLEO collaborations [13]. 
To take into account the off-shellness effect we use for the $\Gamma_{\sigma N N}\left(q^{2}\right)$ vertex a monopole parametrization

$$
\Gamma_{\sigma N N}\left(q^{2}\right)=g_{\sigma N N} F_{\sigma N N}\left(q^{2}\right), \quad F_{\sigma N N}\left(q^{2}\right)=\frac{\Lambda_{\sigma N N}^{2}-m_{\sigma}^{2}}{\Lambda_{\sigma N N}^{2}-q^{2}},
$$

motivated by the models of the nucleon-nucleon interaction [11] with $g_{\sigma N N}=9 \pm 1, \Lambda_{\sigma N N}=2$ $\mathrm{GeV}[11,14]$.

To express the exchange of the wide $\sigma$-meson of mass $m_{\sigma}$ and width $\Gamma_{\sigma}$, we do the following modification of the propagator for the zero-width pole term [15-19]

$$
\frac{1}{m_{\sigma}^{2}-q^{2}} \rightarrow \Delta_{\sigma}\left(q^{2}\right) \equiv \frac{1}{m_{\sigma}^{2}-q^{2}-i m_{\sigma} \Gamma_{\sigma} f\left(q^{2}\right)}=\int_{4 m_{\pi}^{2}}^{\infty} d \lambda \frac{\rho_{\sigma}(\lambda)}{\lambda-q^{2}},
$$

where

and the spectral density is

$$
f\left(q^{2}\right)=\left(\frac{q^{2}-4 m_{\pi}^{2}}{m_{\sigma}^{2}-4 m_{\pi}^{2}}\right)^{1 / 2}
$$

$$
\rho_{\sigma}(\lambda)=\frac{1}{\pi} \frac{\gamma_{\sigma}\left(\lambda-4 m_{\pi}^{2}\right)^{1 / 2}}{\left(\lambda-m_{\sigma}^{2}\right)^{2}+\gamma_{\sigma}^{2}\left(\lambda-4 m_{\pi}^{2}\right)}, \quad \gamma_{\sigma}=\frac{m_{\sigma} \Gamma_{\sigma}}{\left(m_{\sigma}^{2}-4 m_{\pi}^{2}\right)^{1 / 2}} .
$$

Here, $\Gamma_{\sigma}$ denotes the width for decay of the wide $\sigma$-meson into $2 \pi$ state. The density $\rho(\sigma)$ can be interpreted as the mass squared density distribution of the $\sigma$ meson, having the proper threshold (two-pion mass squared), normalization, and width. Further, the exact propagator $\Delta_{\sigma}(4)$ is then well approximated by the sum of two-pole approximation of stable mesons [17-19]

$$
\Delta_{\sigma}\left(q^{2}\right) \approx \frac{A_{1}}{m_{1}^{2}-q^{2}}+\frac{A_{2}}{m_{2}^{2}-q^{2}} .
$$

By fitting left hand side by parametrization of right hand side we find the values of parameters as

$$
\begin{array}{ll}
m_{1}=0.407 \mathrm{GeV}, & A_{1}=0.356, \\
m_{2}=0.985 \mathrm{GeV}, & A_{2}=0.644 .
\end{array}
$$

Finally, the $\sigma \mu \mu$ vertex (see Fig. 1) is

$$
\Gamma_{\sigma \mu \mu}\left(q^{2}\right)=\alpha^{2} m_{\mu} g_{\sigma \gamma \gamma} F_{\sigma \mu \mu}\left(q^{2}\right),
$$

with the $\sigma$-meson-muon form factor being [8]

$$
\begin{gathered}
F_{\sigma \mu \mu}\left(q^{2}\right)=\frac{4}{q^{2}-4 m_{\mu}^{2}} \int \frac{d^{4} l}{i \pi^{2}}\left\{A_{\sigma \gamma \gamma}\left[\frac{1}{l^{2}}-\frac{\left(2 m_{\mu}^{2}+(l, l-q)\right)(l, l-q)}{l^{2}(l-q)^{2}\left((k+l)^{2}-m_{\mu}^{2}\right)}\right]\right. \\
\left.-\frac{1}{2} B_{\sigma \gamma \gamma} q^{2}\left(1-\frac{(l, l-q)}{(k+l)^{2}-m_{\mu}^{2}}\right)\right\},
\end{gathered}
$$

where $A$ and $B$ are two scalar form factors depending on meson $q^{2}$ and photon $q_{1}^{2}=l^{2}, q_{2}^{2}=$ $(l-q)^{2}$ virtualities that define the $\sigma \gamma \gamma$ vertex $[8,20]$

$$
\begin{aligned}
\Gamma_{\mu \nu}^{\sigma \gamma \gamma}\left(q^{2} ; q_{1}^{2}, q_{2}^{2}\right) & =A_{\sigma \gamma \gamma}\left(q^{2} ; q_{1}^{2}, q_{2}^{2}\right)\left(g_{\mu \nu} q_{1} q_{2}-q_{1 \nu} q_{2 \mu}\right) \\
& +B_{\sigma \gamma \gamma}\left(q^{2} ; q_{1}^{2}, q_{2}^{2}\right)\left(q_{1}^{2} q_{2 \mu}-q_{1} q_{2} q_{1 \mu}\right)\left(q_{2}^{2} q_{1 v}-q_{1} q_{2} q_{2 v}\right) .
\end{aligned}
$$


The dispersion representation of the form factor $F_{\sigma \mu \mu}\left(q^{2}\right)$ is $\left(B_{\sigma \gamma \gamma}\right.$ does not contribute to this formula)

$$
F_{\sigma \mu \mu}\left(q^{2}\right)=F_{\sigma \mu \mu}+\frac{q^{2}}{\pi} \int_{0}^{\infty} d s \frac{\operatorname{Im} F_{\sigma \mu \mu}(s)}{s\left(s-q^{2}\right)},
$$

with

$$
\operatorname{Im} F_{\sigma \mu \mu}(s)=\frac{\pi}{\beta} \ln \frac{1-\beta}{1+\beta}, \quad \beta=\sqrt{1-\frac{4 m_{\mu}^{2}}{s}},
$$

and the subtraction constant

$$
\begin{aligned}
& F_{\sigma \mu \mu} \equiv F_{\sigma \mu \mu}(0)=3\left(\ln \xi^{2}-\frac{1}{2}+\int_{0}^{\infty} d t \ln t \frac{\partial A_{\sigma \gamma \gamma}(t, t)}{\partial t}\right) \\
& +2 \xi^{2}\left[\left.\frac{\partial A_{\sigma \gamma \gamma}(t, t)}{\partial t}\right|_{t=0}\left(2 \ln \xi^{2}-\frac{2}{3}\right)+2 \int_{0}^{\infty} d t \ln t \frac{\partial^{2} A_{\sigma \gamma \gamma}(t, t)}{\partial^{2} t}\right]+O\left(\xi^{4}\right),
\end{aligned}
$$

where we introduced the small expansion parameter $\xi^{2}=m_{\mu}^{2} / \Lambda_{\sigma \gamma \gamma}^{2}$. The $\sigma \gamma \gamma$ coupling entering (7) is related to the $\sigma \rightarrow \gamma \gamma$ partial width [21]

$$
g_{\sigma \gamma \gamma}=\sqrt{\frac{4 \Gamma_{\sigma \gamma \gamma}}{\pi \alpha^{2} m_{\sigma}^{3}}}
$$

Using $\Gamma_{\sigma \gamma \gamma}=2.05 \mathrm{keV}$ [22], one gets $g_{\sigma \gamma \gamma}=0.63 \mathrm{GeV}^{-1}$. For $A_{\sigma \gamma \gamma}$ we assume simple expressions motivated by the vector meson dominance models

$$
A_{\sigma \gamma \gamma}^{(1)}\left(q_{1}^{2}, q_{2}^{2}\right)=\frac{\Lambda_{\sigma \gamma \gamma}^{2}}{\Lambda_{\sigma \gamma \gamma}^{2}-q_{1}^{2}-q_{2}^{2}}, \quad A_{\sigma \gamma \gamma}^{(2)}\left(q_{1}^{2}, q_{2}^{2}\right)=\frac{\Lambda_{\sigma \gamma \gamma}^{4}}{\left(\Lambda_{\sigma \gamma \gamma}^{2}-q_{1}^{2}\right)\left(\Lambda_{\sigma \gamma \gamma}^{2}-q_{2}^{2}\right)},
$$

with $\Lambda_{\sigma \gamma \gamma}^{2}=0.6 \mathrm{GeV}^{2}$. The first form factor is in agreement with QCD asymptotics, while the second one is often used in experimental studies. Then we get for the subtraction constant in $(10)$

$$
\begin{aligned}
& F_{\sigma \mu \mu}^{(1)}=-\left[3 \ln \left(2 \xi^{2}\right)+8 \xi^{2}\left(\ln \left(2 \xi^{2}\right)+\frac{2}{3}\right)\right]+O\left(\xi^{4}\right) \approx 10.34 \\
& F_{\sigma \mu \mu}^{(2)}=-\left[3 \ln \xi^{2}+\frac{3}{2}+8 \xi^{2}\left(\ln \left(\xi^{2}\right)+\frac{7}{6}\right)\right]+O\left(\xi^{4}\right) \approx 10.84 .
\end{aligned}
$$

We see, that due to large logarithm contributions $\ln \left(\xi^{2}\right)$, there is almost no sensitivity to the choice of $\sigma \gamma \gamma$ form factor in (14).

The next step is the essential one in the Breit potential derivation: we perform the semirelativistic expansion $q^{2} \approx-\boldsymbol{q}^{2}, q_{1,2}^{2} \approx-Q_{1,2}^{2}$. In doing the expansion of (1), we shall neglect the dependence of the denominator on the photon energy, i.e., neglect retardation effects

$$
V_{\sigma}(\boldsymbol{q})=\lambda_{\sigma} F_{\sigma N N}\left(-\boldsymbol{q}^{2}\right)\left[F_{\sigma \mu \mu}-\frac{\boldsymbol{q}^{2}}{\pi} \int_{0}^{\infty} d s \frac{\operatorname{Im} F_{\sigma \mu \mu}(s)}{s\left(s+\boldsymbol{q}^{2}\right)}\right] \sum_{i=1,2} \frac{A_{i}}{m_{i}^{2}+\boldsymbol{q}^{2}}
$$

with

$$
\lambda_{\sigma}=\alpha^{2} m_{\mu} g_{\sigma \gamma \gamma} g_{\sigma N N} .
$$

By using the algebraic relation

$$
\frac{1}{\boldsymbol{q}^{2}+A} \frac{1}{\boldsymbol{q}^{2}+B}=\frac{1}{B-A}\left(\frac{1}{\boldsymbol{q}^{2}+A}-\frac{1}{\boldsymbol{q}^{2}+B}\right)
$$


it is easy to transform (16) to the form linear in $1 /\left(\boldsymbol{q}^{2}+A\right)$ with its Fourier transform

$$
\frac{1}{\boldsymbol{q}^{2}+a^{2}} \stackrel{F . t .}{\Longrightarrow} \frac{1}{4 \pi} \frac{1}{r} e^{-a r}
$$

If we restrict ourself for the moment by the leading contribution $\left(\Lambda_{\sigma N N}^{2} \rightarrow \infty, \operatorname{Im} F_{\sigma \mu \mu}(s) \rightarrow 0\right)$

$$
V_{\sigma}^{(0)}(\boldsymbol{q})=\lambda_{\sigma} F_{\sigma \mu \mu}(0) \sum_{i=1,2} \frac{A_{i}}{m_{i}^{2}+\boldsymbol{q}^{2}},
$$

the coordinate-space potential will be

$$
V_{\sigma}^{(0)}(\boldsymbol{r})=\frac{\lambda_{\sigma}}{4 \pi} F_{\sigma \mu \mu} \sum_{i=1,2} A_{i} \frac{1}{r} e^{-m_{i} r} .
$$

Averaging (18) over the wave functions of 1S-, 2S- and 2P-states by using

$$
\begin{aligned}
\left\langle 1 S\left|\frac{1}{r} e^{-m_{i} r}\right| 1 S\right\rangle & =\frac{4 w \widetilde{w}_{i}^{2}}{\left(1+2 \widetilde{w}_{i}\right)^{2}},\left\langle 2 S\left|\frac{1}{r} e^{-m_{i} r}\right| 2 S\right\rangle=\frac{w \widetilde{w}_{i}^{2}\left(2+\widetilde{w}_{i}^{2}\right)}{4\left(1+\widetilde{w}_{i}\right)^{4}}, \\
\left\langle 2 P\left|\frac{1}{r} e^{-m_{i} r}\right| 2 P\right\rangle & =\frac{w \widetilde{w}_{i}^{4}}{4\left(1+\widetilde{w}_{i}\right)^{4}},
\end{aligned}
$$

where $m_{r}=m_{N} m_{\mu} /\left(m_{N}+m_{\mu}\right), w=\alpha m_{r}, \widetilde{w}_{i}=w / m_{i}$, we obtain the energy shifts

$$
\begin{aligned}
E_{\sigma}^{(0)}(1 S) & =-\frac{\lambda_{\sigma}}{\pi} F_{\sigma \mu \mu} \sum_{i=1,2} A_{i} \frac{w \widetilde{w}_{i}^{2}}{\left(1+2 \widetilde{w}_{i}\right)^{2}}, \\
E_{\sigma}^{(0)}(2 S) & =-\frac{\lambda_{\sigma}}{16 \pi} F_{\sigma \mu \mu} \sum_{i=1,2} A_{i} \frac{w \widetilde{w}^{2}\left(2+\widetilde{w}_{i}^{2}\right)}{\left(1+\widetilde{w}_{i}\right)^{4}}, \\
E_{\sigma}^{(0)}(2 P) & =\frac{\lambda_{\sigma}}{16 \pi} \sum_{i=1,2} A_{i}\left[F_{\sigma \mu \mu}-\frac{m_{i}^{2}}{4 m_{\mu}^{2}}\left(1+\frac{4}{3} \widetilde{w}_{i}+\frac{1}{2} \widetilde{w}_{i}^{2}\right) \widetilde{F}_{\sigma \mu \mu}\right] \frac{w \widetilde{w}_{i}^{4}}{\left(1+\widetilde{w}_{i}\right)^{4}},
\end{aligned}
$$

where

$$
\widetilde{F}_{\sigma \mu \mu}^{(2)}=-\left[\frac{3}{2}+2 \xi^{2}\left(\ln \xi^{2}+\frac{5}{3}\right)\right] .
$$

Two expressions (21), (22) give contributions which are different in sign just as in the case of pseudoscalar meson. The result for the lowest levels is

$$
\begin{aligned}
& \Delta E_{1 S}=-0.110 \mathrm{meV}, \\
& \Delta E_{2 S}=-0.014 \mathrm{meV}, \\
& \Delta E_{2 P}=2.6 \cdot 10^{-8} \mathrm{meV},
\end{aligned}
$$

They contribute to the Lamb shift $(2 \mathrm{P}-2 \mathrm{~S})$

$$
\Delta E_{\text {LambShift }}^{\sigma}(2 \mathrm{P}-2 \mathrm{~S})=0.014 \mathrm{meV} \text {. }
$$

\section{Conclusion}

Our result for $\sigma$-meson is in agreement with previously obtained estimates in [8, 23]. The obtained contributions of scalar mesons to the Lamb shift (2P-2S) in muonic hydrogen are large and should be used for precise comparison with experimental data of CREMA collaboration $[1,2]$. 


\section{Acknowledgment}

The work is supported by Russian Science Foundation (grant No. RSF 18-12-00128).

\section{References}

[1] A. Antognini et al., Ann. Phys. (NY) 331, 127 (2013)

[2] M. Diepold et al., Ann. Phys. (NY) 396, 220 (2018)

[3] P.J. Mohr, B.N. Taylor and D.B. Newell, Rev. Mod. Phys. 88, 035009 (2016)

[4] V.M. Budnev et al., Phys. Rep. 15, 181 (1975)

[5] N.T. Huong, E. Kou, B. Moussallam, Phys. Rev. D9, 114005 (2016)

[6] F. Hagelstein and V. Pascalutsa, PoS CD15, 077 (2016)

[7] H.Q. Zhou and H.R. Pang, Phys. Rev. A92, 032512 (2015)

[8] D. Borisyuk, Phys. Rev. C96, 055201 (2017)

[9] A.E. Dorokhov, N.I. Kochelev, A.P. Martynenko et al., Phys. Part. Nucl. Lett. 14, 857 (2017)

[10] A.E. Dorokhov, N.I. Kochelev, A.P. Martynenko et al., Phys. Lett. B776, 105 (2018)

[11] R. Machleidt, K. Holinde and C. Elster, Phys. Rept. 149, 1 (1987)

[12] E.M. Aitala et al., [E791 Collaboration] Phys. Rev. Lett. 86, 770 (2001)

[13] H. Muramatsu et al., [CLEO Collaboration] Phys. Rev. Lett. 89, 251802 (2002)

[14] A. Calle Cordon, E. Ruiz Arriola, Phys. Rev. C81, 044002 (2010)

[15] P. Curry, J.W. Moffat, Phys. Rev. 184, 1885 (1969)

[16] V. Wataghin, Nucl. Phys. B10 107 (1968)

[17] J. Binstock, R. Bryan, Phys. Rev. D4, 1341 (1971)

[18] R. Bryan, A. Gersten, Phys. Rev. D6, 341 (1972)

[19] V.G.J. Stoks, R.A.M. Klomp, C.P.F. Terheggen and J.J. de Swart, Phys. Rev. C49, 2950 (1994)

[20] A.E. Dorokhov, A.E. Radzhabov, and A.S. Zhevlakov, EPJ C72, 2227 (2012)

[21] A. Faessler, T. Gutsche, M.A. Ivanov, V.E. Lyubovitskij, P. Wang, Phys. Rev. D68, $014011(2003)$

[22] L.Y. Dai and M.R. Pennington, Phys. Lett. B736, 11 (2014)

[23] A. E. Dorokhov, A. P. Martynenko, F. A. Martynenko and A. E. Radzhabov, EPJ Web Conf. 204 (2019) 05008. 